\title{
Neoclassical impurity flux in presence of turbulent generated poloidal asymmetries and pressure anisotropy
}

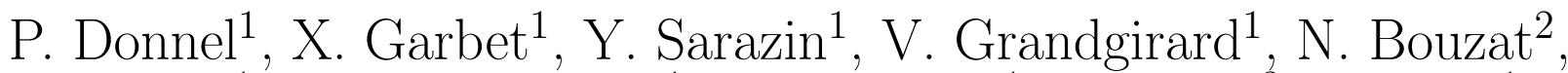 \\ E.Caschera ${ }^{1}$, G. Dif-Pradalier ${ }^{1}$, P. Ghendrih ${ }^{1}$, C. Gillot ${ }^{3}$, G. Latu${ }^{1}$, C. \\ Passeron $^{1}$
}

\begin{abstract}
Poloidal asymmetries of impurities are commonly observed experimentally. Density asymmetry is already known to impact significantly neoclassical prediction of impurity flux. In this article, the effect of impurity pressure asymmetry and anisotropy on the neoclassical flux of impurity is derived analytically. This prediction is compared with results coming from a simulation performed with the gyrokinetic code GYSELA, featuring both turbulent and neoclassical transports. A fair agreement is found between the analytical prediction and the result of the simulation. On the special case which is considered, the effect of impurity pressure asymmetry and anisotropy are shown to play a predominant role on the neoclassical impurity transport.
\end{abstract}

\section{Introduction}

Impurity transport is an issue of utmost importance for fusion. One reason is the choice of tungsten for the ITER divertor. Indeed high-Z materials are only partially ionized in the plasma core, so that they may lead to prohibitive radiative losses even at low concentrations, and impact dramatically plasma performance and stability. On-axis accumulation of tungsten has been widely observed in tokamaks $[1,2]$. In current tokamaks, neoclassical transport is dominant for heavy impurities due to a large collisionality of these ions and the presence of poloidal asymmetry of the impurity density induced by ICRH and NBI sources [3]. These asymmetries are known to modify significantly (up to an order of magnitude) neoclassical flux of impurity [4]. ITER is expected to use ECRH heating system which should reduce the externally imposed poloidal asymmetry of impurity density. In this context, one may wonder whether poloidal asymmetries induced by turbulence [5] play an important role in impurity transport. Up to recently, first principles simulations of turbulent and neoclassical fluxes were performed with different dedicated codes, implicitly assuming that both transport channels are separable and therefore additive. One of the key questions is whether this assumption is valid.

Preliminary simulations obtained with the gyrokinetic code GYSELA [6] have shown evidence of a neoclassical-turbulence synergy for impurity transport [7]. However no clear theoretical explanation was given, although poloidal asymmetries were pointed out as critical players. New simulations have been done using a new and more accurate collision operator [8] and improved boundary conditions [9]. The new collision operator allows in particular the isotropisation of the pressure thanks to the inclusion of derivatives with respect to the adiabatic invariant $\mu$. This version of GYSELA has been successfully benchmarked against neoclassical theory. In particular, analytical predictions of the pinch velocity and screening factor are recovered.

The new simulations confirm the neoclassical-turbulence synergy and allow identification of a mechanism that underlie this synergy. The new simulations have indeed shown strong poloidal asymmetries attributed to the presence of turbulence. Poloidal asymmetries of the impurity density is known to impact significantly the neoclassical flux of impurity $[4,10,11]$. In this article a generalization of this approach is performed by including the effect of pressure anisotropy and poloidal 
asymmetries in the neoclassical prediction of impurity flux. This new prediction is then convincingly compared to results coming from the GYSELA code. It is stressed that pressure asymmetries can lead to a significant modification of the neoclassical flux of impurities.

\section{Neoclassical impurity flux in presence of large poloidal asymmetries and pressure anisotropy}

In current tokamaks, heavy impurities like tungsten are in the Pfirsch-Schlüter regime. But on larger devices, like ITER, heavy impurities should be closer to the plateau regime due to higher temperatures. The banana-plateau contribution of the impurity flux is expected to be linked to the pressure anisotropy. Moreover, the poloidal asymmetry of the impurity density is known to modify significantly (up to an order of magnitude) neoclassical flux of impurity in the Pfirsch-Schlüter regime [4]. It is therefore natural to take into account both pressure anisotropy and poloidal asymmetries of the impurity for the computation of the neoclassical flux of impurities.

The computation of the neoclassical impurity flux in this framework is done in three steps. First, the perpendicular flow is expressed. An unknown function $K_{z}(\psi, \theta)$, related to the impurity poloidal velocity, is then determined using the incompressibility of the flow and parallel force balance. It leads to the expression of the impurity flux.

\subsection{Structure of the impurity flow in presence of poloidal asymmetries}

For the main ion species, it is assumed that there are no changes compared with the standard neoclassical theory. In other words, poloidal asymmetries and anisotropy of the main ion species are at their neoclassical level which is small $[12,13]$. On the other hand, large poloidal asymmetries and anisotropy are allowed for the impurity.

The parallel velocity of the impurity is required to compute the neoclassical flux. Its computation is made in two steps. First the perpendicular flux of the impurity is computed. It is then link to the parallel flux using the incompressibility of the flow which is imposed by the stationarity assumption.

To compute the perpendicular flux of impurity, the idea is to derive a vectorial expressions of particle and heat fluxes. This is not obvious because of the scalar form of the gyrokinetic equation, i.e.

$$
\partial_{t} \bar{F}_{z}+\frac{1}{B_{\| z}^{*}} \nabla_{\mathbf{z}} \cdot\left(\dot{\mathbf{z}} B_{\| z}^{*} \bar{F}_{z}\right)=C\left(\bar{F}_{z}\right)
$$

where $\bar{F}_{z}$ is the distribution function of the impurity which is considered, $C$ is the collision operator and $B_{\| z}^{*}=B+\frac{m_{z}}{Z e} v_{\|} \boldsymbol{b} \cdot(\boldsymbol{\nabla} \times \boldsymbol{b})$, with $\mathbf{b}=\frac{\mathbf{B}}{B}$, corresponds to the Jacobian of the gyrocenter system of coordinates. It must be kept in mind that the actual trajectories of particles differ from those of gyrocenters by a cyclotron motion. The limit of large scale flows is now considered. It is well described by the drift kinetic limit $k_{\perp} \rho_{z} \rightarrow 0$ of the gyrokinetic equation Eq.(1). At first order in $\rho_{\star z}=\frac{\rho_{z}}{a}$ the particle velocity is of the form $\mathbf{v}=v_{\|} \mathbf{b}+\mathbf{v}_{E}+\mathbf{v}_{D}$, where $\mathbf{v}_{E}$ is the $E \times B$ drift velocity and $\mathbf{v}_{D}$ is the sum of curvature and $\nabla B$ drift velocities. Taking the first moment of Eq.(1), one gets $\nabla \cdot \boldsymbol{\Gamma}_{z}=0$ in steady-state, where $\boldsymbol{\Gamma}_{z}=\Gamma_{\| z} \mathbf{b}+\Gamma_{\perp z}$. Note however that this is a flux of guiding centers. One needs the particle flux. This is done by adding the magnetization flux, whose divergence is zero and does not change the conservative form $\nabla \cdot \Gamma_{z}=0$. Hence

$$
\Gamma_{\perp z}=N_{z} \mathbf{v}_{E}+N_{z}\left\langle\mathbf{v}_{D}\right\rangle_{v}-\nabla \times\left[N_{z}\left\langle\frac{\mu}{Z e} \mathbf{b}\right\rangle_{v}\right]
$$

where $\mu=\frac{m_{z} v_{\perp}^{2}}{2 B}$ is the adiabatic invariant and the bracket is an average over the distribution function.

$$
\langle\ldots\rangle_{v}=\frac{1}{N_{z}} \int d^{3} \mathbf{v} \bar{F}_{z} \ldots
$$


The perpendicular flow associated with the curvature drift plus magnetization term is

$$
\boldsymbol{\Gamma}_{\perp}^{D}=N_{z}\left\langle\mathbf{v}_{D}\right\rangle_{v}=\frac{\mathbf{b}}{Z e B} \times\left[\nabla P_{\perp z}+\Pi_{\| z} \boldsymbol{\kappa}\right]
$$

where $\boldsymbol{\kappa}=-\mathbf{b} \times(\nabla \times \mathbf{b})$ is the field curvature, $\Pi_{\| z}=P_{\| z}-P_{\perp z}$ represents the pressure anisotropy, with $P_{\| z}=\int d^{3} \mathbf{v} \bar{F}_{z} m_{z} v_{\|}^{2}$ and $P_{\perp z}=\int d^{3} \mathbf{v} \bar{F}_{z} \mu B$. This perpendicular flux $\boldsymbol{\Gamma}_{\perp}^{D}$ can be reexpressed as the divergence of a CGL stress tensor

$$
\Pi_{z, i j}=\int d^{3} \mathbf{v} \bar{F}_{z} m_{z} v_{i} v_{j}=P_{\| z} b_{i} b_{j}+P_{\perp z}\left(\delta_{i j}-b_{i} b_{j}\right)
$$

by noting that

$$
\nabla \cdot \boldsymbol{\Pi}_{z}=\nabla P_{\perp z}+\Pi_{\| z} \boldsymbol{\kappa}+\left[(\mathbf{B} \cdot \nabla)\left(\frac{\Pi_{\| z}}{B}\right)\right] \mathbf{b}
$$

so that

$$
\boldsymbol{\Gamma}_{z}=\Gamma_{\| z} \mathbf{b}+N_{z} \frac{\mathbf{b}}{B} \times \nabla \phi+\frac{\mathbf{b}}{Z e B} \times \nabla \cdot \boldsymbol{\Pi}_{z}
$$

Noting that for an axisymmetric problem, $\nabla \phi=\frac{\partial \phi}{\partial \psi} \nabla \psi+\frac{\partial \phi}{\partial \theta} \nabla \theta$ and $\nabla P_{\perp z}=\frac{\partial P_{\perp z}}{\partial \psi} \nabla \psi+\frac{\partial P_{\perp z}}{\partial \theta} \nabla \theta$, and using the identity

$$
\frac{\mathbf{b}}{B} \times \nabla \psi=I \frac{\mathbf{b}}{B}-R^{2} \nabla \varphi
$$

where $I$ is the current and $R$ the major radius, one can express the impurity flux as follow

$$
\boldsymbol{\Gamma}_{z}=K_{z} \mathbf{B}-N_{z} \Omega_{z} R^{2} \nabla \varphi+\frac{\Pi_{\| z}}{Z e B}(\mathbf{b} \times \boldsymbol{\kappa})+\left(N_{z} \frac{\partial \phi}{\partial \theta}+\frac{1}{Z e} \frac{\partial P_{\perp z}}{\partial \theta}\right) \frac{\mathbf{b}}{B} \times \nabla \theta
$$

All quantities depend on $(\psi, \theta)$ and the following definitions have been introduced

$$
\begin{gathered}
\Omega_{z}=\frac{\partial \phi}{\partial \psi}+\frac{1}{N_{z} Z e} \frac{\partial P_{\perp z}}{\partial \psi} \\
K_{z}=\frac{\Gamma_{\| z}}{B}+\frac{I}{B^{2}} N_{z} \Omega_{z}
\end{gathered}
$$

\subsection{Determination of the poloidal variation of $K_{z}$}

The poloidal variation of $K_{z}(\psi, \theta)$ is constrained by the incompressibility of the flow $\nabla \cdot \boldsymbol{\Gamma}_{z}=0$, which reads

$$
\mathbf{B} \cdot \nabla K_{z}+\nabla \cdot \tilde{\boldsymbol{\Gamma}}_{z}=0
$$

where

$$
\tilde{\boldsymbol{\Gamma}}_{z}=\frac{\Pi_{\| z}}{Z e B}(\mathbf{b} \times \boldsymbol{\kappa})+\left(N_{z} \frac{\partial \phi}{\partial \theta}+\frac{1}{Z e} \frac{\partial P_{\perp z}}{\partial \theta}\right) \frac{\mathbf{b}}{B} \times \nabla \theta
$$

The expression of the divergence of a vector $\boldsymbol{V}$ that does not depend on $\varphi$ is

$$
\frac{\nabla \cdot \boldsymbol{V}}{\mathbf{B} \cdot \nabla \theta}=\frac{\partial}{\partial \psi}\left(\frac{\boldsymbol{V} \cdot \nabla \psi}{\mathbf{B} \cdot \nabla \theta}\right)+\frac{\partial}{\partial \theta}\left(\frac{\boldsymbol{V} \cdot \nabla \theta}{\mathbf{B} \cdot \nabla \theta}\right)
$$

Using the following relations

$$
\begin{aligned}
(\mathbf{b} \times \boldsymbol{\kappa}) \cdot \nabla \psi & =I \mathbf{B} \cdot \nabla \theta \frac{\partial}{\partial \theta}\left(\frac{1}{B}\right) \\
(\mathbf{b} \times \boldsymbol{\kappa}) \cdot \nabla \theta & =-I \mathbf{B} \cdot \nabla \theta \frac{\partial}{\partial \psi}\left(\frac{1}{B}\right) \\
(\mathbf{B} \times \nabla \theta) \cdot \nabla \psi & =-I \mathbf{B} \cdot \nabla \theta
\end{aligned}
$$


one then obtains

$$
\frac{\nabla \cdot \tilde{\boldsymbol{\Gamma}}_{z}}{\mathbf{B} \cdot \nabla \theta}=\frac{\partial}{\partial \theta}\left[\frac{1}{B} \frac{\partial}{\partial \psi}\left(\frac{I \Pi_{\| z}}{Z e B}\right)\right]-\frac{\partial}{\partial \psi}\left\{\frac{I}{Z e B^{2}}\left[N_{z} Z e \frac{\partial \phi}{\partial \theta}+\frac{\partial P_{\perp z}}{\partial \theta}+B \frac{\partial}{\partial \theta}\left(\frac{\Pi_{\| z}}{B}\right)\right]\right\}
$$

At this stage, one can note that in the absence of poloidal asymmetries $\left(\partial_{\theta}=0\right)$ and pressure anisotropy $\left(\Pi_{\| z}=0\right), \nabla \cdot \tilde{\boldsymbol{\Gamma}}_{z}=0$. Therefore $K_{z}$ depends on $\psi$ only as in the literature [4]. For low Mach number, the parallel force balance reads

$$
\mathbf{B} \cdot \nabla \theta\left[N_{z} Z e \frac{\partial \phi}{\partial \theta}+\frac{\partial P_{\perp z}}{\partial \theta}+B \frac{\partial}{\partial \theta}\left(\frac{\Pi_{\| z}}{B}\right)\right]=B R_{\| z i}
$$

Then putting together Eq.(5), (6) and (7) an equation for $K_{z}$ can be derived

$$
\frac{\partial}{\partial \theta}\left[K_{z}+\frac{1}{B} \frac{\partial}{\partial \psi}\left(\frac{I \Pi_{\| z}}{Z e B}\right)\right]=\frac{\partial}{\partial \psi}\left[\frac{I R_{\| z i}}{Z e B(\mathbf{B} \cdot \nabla \theta)}\right]
$$

Note that an integration on $\theta$ of Eq.(8) leads to $\frac{\partial \Gamma_{z, \text { neo }}^{\psi}}{\partial \psi}=0$, where the neoclassical impurity flux is given by

$$
\Gamma_{z, \text { neo }}^{\psi}=-\frac{I}{Z e}\left\langle\frac{R_{\| z i}}{B}\right\rangle_{\psi}
$$

and the bracket denotes the flux surface average:

$$
\langle f\rangle_{\psi}=\frac{\oint \frac{d \theta d \varphi}{\mathbf{B} \cdot \nabla \theta} f}{\oint \frac{d \theta d \varphi}{\mathbf{B} \cdot \nabla \theta}}
$$

The condition $\frac{\partial \Gamma_{z, \text { neo }}^{\psi}}{\partial \psi}=0$ may be surprising at first sight. But on second thoughts, this condition is natural. Indeed, in the approach considered, there is no source of particles, no turbulence and a steady state solution is considered. The neoclassical flux of impurity is therefore equal to a constant. To alleviate this conceptual paradox, one should keep in mind that time derivatives are neglected in the neoclassical theory because they typically scale as the inverse of the confinement time which is much larger than any other typical time of the problem. But rigorously, time derivatives are not equal to zero.

Unfortunately, the analytical solution of Eq.(8) is not trivial as $R_{\| z i}$ is an implicit function of $K_{z}$. To continue the analytical derivation, the rhs of Eq.(8) is neglected. This is the equivalent of neglecting locally the collisional contribution in the parallel force balance Eq.(7). It can be shown that this approximation is valid if $Z \sqrt{\epsilon} q \nu_{\star, i} \ll 1$ where $\epsilon=\frac{r}{R_{0}}$ is the inverse aspect ratio, $q$ the safety factor and $\nu_{\star, i}$ the collisionality of the main ion species. This approximation is therefore valid for light impurities or if the collisionality of the main ion species is low enough. Note that even though the collision friction is neglected locally in the parallel force balance Eq.(7), it has to be kept in its flux surface average version, for the expression of the neoclassical flux. It is quite remarkable that no solubility problem arises when the friction force is neglected locally in Eq.(7). The function $K_{z}$ then reads $K_{z}(\psi, \theta)=K_{z 0}(\psi)+K_{z 1}(\psi, \theta)$, where

$$
\begin{gathered}
K_{z 0}=\left\langle K_{z}\right\rangle_{\psi} \\
K_{z 1}=-\frac{1}{B} \frac{\partial}{\partial \psi}\left(\frac{I \Pi_{\| z}}{Z e B}\right)+\left\langle\frac{1}{B} \frac{\partial}{\partial \psi}\left(\frac{I \Pi_{\| z}}{Z e B}\right)\right\rangle_{\psi}
\end{gathered}
$$

\subsection{Neoclassical flux}

In the case of a trace $\left(\frac{N_{z} Z^{2}}{N_{i}} \ll 1\right)$ and heavy impurity $\left(\frac{m_{z}}{m_{i}} \gg 1\right)$, it can be shown that the friction force reads (proof is in appendix)

$$
R_{\| z i}=m_{z} \nu_{z i}\left\{-N_{Z} \frac{T_{i}}{e B} \frac{I}{L_{\psi}}+B\left(N_{z} u-K_{z}\right)\right\}
$$


where $\frac{1}{L_{\psi}}=\frac{1}{L_{\psi, i}}+\frac{1}{L_{\psi, z}}$ with $\frac{1}{L_{\psi, i}}=\frac{\partial \ln P_{i}}{\partial \psi}-\frac{3}{2} \frac{\partial \ln T_{i}}{\partial \psi}$ and $\frac{1}{L_{\psi, z}}=-\frac{1}{T_{i} Z N_{z}} \frac{\partial P_{\perp z}}{\partial \psi} . L_{\psi, i}$ is a flux function whereas $L_{\psi, z}$ is a function of $\psi$ and $\theta$. In Eq.(11) $u$ is a flux function given by

$$
u=\left(k_{n e o}-\frac{3}{2}\right) \frac{I}{e\left\langle B^{2}\right\rangle_{\psi}} \frac{\partial T_{i}}{\partial \psi}
$$

This quantity is closely link to the poloidal rotation of the main ion species as can be seen from the presence of $k_{\text {neo }}$ in its expression. If the main ion is in the banana regime and large aspect ratio are considered $\epsilon \ll 1$, then $k_{n e o} \simeq 1.17$ and $u$ takes the limit given in the literature [14] $\simeq \simeq-0.33 \frac{I}{e\left\langle B^{2}\right\rangle} \frac{\partial T_{i}}{\partial \psi}$. This value is used in the following. Note that $\nu_{z i}$ is also a flux function. Using Eq.(7), while keeping the friction force leads to the solubility constraint

$$
\left\langle\frac{B R_{\| z i}}{N_{z}}\right\rangle_{\psi}=\left\langle\frac{\mathbf{B} \cdot \nabla \theta}{N_{z}}\left[\frac{\partial P_{\perp z}}{\partial \theta}+B \frac{\partial}{\partial \theta}\left(\frac{\Pi_{\| z}}{B}\right)\right]\right\rangle_{\psi}
$$

Combining Eq.(10), Eq.(11) and Eq.(13), one finds

$$
\begin{aligned}
K_{z 0} & =-\frac{T_{i} I}{e}\left\langle\frac{1}{L_{\psi}}\right\rangle_{\psi}\left\langle\frac{B^{2}}{N_{Z}}\right\rangle_{\psi}^{-1}+u\left\langle B^{2}\right\rangle_{\psi}\left\langle\frac{B^{2}}{N_{z}}\right\rangle_{\psi}^{-1} \\
& +\left\langle\frac{B}{N_{z}} \frac{\partial}{\partial \psi}\left(\frac{I \Pi_{\| z}}{Z e B}\right)\right\rangle_{\psi}\left\langle\frac{B^{2}}{N_{z}}\right\rangle_{\psi}^{-1}-\left\langle\frac{1}{B} \frac{\partial}{\partial \psi}\left(\frac{I \Pi_{\| z}}{Z e B}\right)\right\rangle_{\psi} \\
& -\frac{1}{m_{z} \nu_{z i}}\left\langle\frac{\mathbf{B} \cdot \nabla \theta}{N_{z}}\left[\frac{\partial P_{\perp z}}{\partial \theta}+B \frac{\partial}{\partial \theta}\left(\frac{\Pi_{\| z}}{B}\right)\right]\right\rangle_{\psi}\left\langle\frac{B^{2}}{N_{z}}\right\rangle_{\psi}^{-1}
\end{aligned}
$$

This expression of $K_{z 0}$ can then be plugged into the friction force to calculate the neoclassical impurity radial flux Eq.(9):

$$
\begin{aligned}
& \Gamma_{z, \text { nео }}^{\psi}=\Gamma_{z, B P}^{\psi}+\Gamma_{z, P S}^{\psi} \\
& \Gamma_{z, B P}^{\psi}=-\frac{I}{Z e} \frac{1}{\left\langle\frac{B^{2}}{N_{z}}\right\rangle_{\psi}}\left\langle\frac{\mathbf{B} \cdot \nabla \theta}{N_{z}}\left[\frac{\partial P_{\perp z}}{\partial \theta}+B \frac{\partial}{\partial \theta}\left(\frac{\Pi_{\| z}}{B}\right)\right]\right\rangle_{\psi} \\
& \Gamma_{z, P S}^{\psi}=\frac{I}{Z e} m_{z} \nu_{z i}\left\{\frac{T_{i}}{e} \frac{I}{L_{\psi, i}}\left(\left\langle\frac{N_{z}}{B^{2}}\right\rangle_{\psi}-\frac{1}{\left\langle\frac{B^{2}}{N_{z}}\right\rangle_{\psi}}\right)-u\left(\left\langle N_{z}\right\rangle_{\psi}-\frac{\left\langle B^{2}\right\rangle_{\psi}}{\left\langle\frac{B^{2}}{N_{z}}\right\rangle_{\psi}}\right)\right. \\
& \left.+\frac{T_{i} I}{e}\left(\left\langle\frac{N_{z}}{B^{2} L_{\psi, z}}\right\rangle_{\psi}-\left\langle\frac{1}{L_{\psi, z}}\right\rangle_{\psi} \frac{1}{\left\langle\frac{B^{2}}{N_{z}}\right\rangle_{\psi}}\right)-\left\langle\frac{1}{B} \frac{\partial}{\partial \psi}\left(\frac{I \Pi_{\| z}}{Z e B}\right)\right\rangle_{\psi}+\frac{\left\langle\frac{B}{N_{z}} \frac{\partial}{\partial \psi}\left(\frac{I \Pi_{\| z}}{Z e B}\right)\right\rangle_{\psi}}{\left\langle\frac{B^{2}}{N_{z}}\right\rangle_{\psi}}\right\}
\end{aligned}
$$

Eq.(16) can be seen as a modification of the banana-plateau flux by poloidal asymmetries. Note that it is independent of the interspecies collisionality and therefore dominates for low collisionality regimes.

The first and second terms of Eq.(17) are identical to Eq.(10) of [4] for which only poloidal density asymmetries are considered. The third term corresponds to a simple diffusion which is often neglected but can become important when impurity peaking is strong. The last two terms are entirely controlled by $K_{z 1}$, and represent a modification of the friction force due to the impurity pressure anisotropy. 


\section{Comparison with results from gyrokinetic simulations}

The goal of this section is to assess the relative importance of the various contributions in the neoclassical prediction derived in the previous section Eq.(15) using the results of a simulation performed with the GYSELA code.

\subsection{Choice of the simulation parameters}

For this simulation, the main ion species is deuterium and the impurity is tungsten with a fixed charge state $(Z=40)$ in the trace limit $\left(\frac{N_{z} Z^{2}}{N_{i}} \sim 10^{-3}\right)$. The electron response is adiabatic. The size of the machine simulated is fixed by the dimensionless parameter $\rho_{\star i}=\frac{\rho_{i}}{a}=\frac{1}{190}$ where $\rho_{i}$ is the Larmor radius of a thermal ion at mid-radius. GYSELA uses a simplified geometry with circular concentric magnetic surfaces characterized by an inverse aspect ratio $\frac{R_{0}}{a}=4.4$ and a safety factor profile $q(r)=1.5+1.3 \exp \left[2.5 \log \left(\frac{r}{a}\right)\right]$. The two ion species are initiated with identical density and temperature profiles $\frac{d \ln N_{s}(r)}{d r}=-2.2 \cosh ^{-2}\left[25\left(\frac{r}{a}-0.5\right)\right]$ and $\frac{d \ln T_{s}(r)}{d r}=-6 \cosh ^{-2}\left[25\left(\frac{r}{a}-0.5\right)\right]$. To approach a statistical steady state, an isotropic source of energy is added [6]. Its amplitude depends only on the radius. Finally, the outer boundary condition is ensured via a penalization technique [9]. More precisely, a krook term with a radial dependence is applied on the distribution function. It allows to extract the energy coming from the heat flux without injecting/removing particles.

The time step of the simulation is $\Delta t \omega_{c i}=16$ and is chosen to resolve accurately both turbulent and collisional time scales. A high spatial resolution is needed bacause the Larmor radius of the impurity is smaller than the one of the main ion $\rho_{z}=\frac{1}{Z} \sqrt{\frac{m_{z}}{m_{i}}} \rho_{i}$. Because of this high resolution and the presence of two species, the numerical cost of this simulation is very large. In order to reduce this cost, the following strategy has been used: in a first stage, the code is first run without impurity until statistical steady-state with a resolution of $\left(N_{r}, N_{\theta}, N_{\varphi}, N_{v_{\|}}, N_{\mu}\right)=(256,512,32,127,64)$ which is enough for the main ion species. A second step is then performed, with a resolution increased in view of the later impurity introduction $\left(N_{r}, N_{\theta}, N_{\varphi}, N_{v_{\|}}, N_{\mu}\right)=(512,1024,32,127,64)$. This step, performed without impurities, is done to allow for some reorganization of the system when numerical resolution is increased. Eventhough this reorganization is rather short, it is numerically cheaper to perform it in absence of impurities. Finally, the impurity is added with the same resolution $\left(N_{r}, N_{\theta}, N_{\varphi}, N_{v_{\|}}, N_{\mu}\right)=(512,1024,32,127,64)$ when statistical steady-state is reached without impurities. Despite this strategy, the convergence of a simulation with impurity toward a statistical steady-state still requires several millions of CPU hours.

It has therefore been decided to focus on a single well resolved simulation for a quantitative comparison between theory and simulation. In GYSELA, the collisionality of the main ion species at mid-radius $\nu_{i}^{\star}\left(\frac{r}{a}=0.5\right)$ is a free parameter. For an efficient test of the theory, the collisionality of the main ion species has been fixed at $\nu_{i}^{\star}\left(\frac{r}{a}=0.5\right)=10^{-1}$. Indeed with this choice, the main ion species is in the banana regime in the whole domain whereas the collisionality of the impurity is close to the plateau/Pfirsch-Schlüter transition allowing to test both the banana/plateau Eq.(16) and the Pfirsch-Schlüter Eq.(17) predictions with a single simulation. The collisionality of tungsten in the considered simulation is given in the Fig.1. The impurity is in the Pfirsch-Schlüter regime in all the simulation domain but in the region ranging between $0.15 \leq \frac{r}{a} \leq 0.4$ where it is close to the plateau regime.

Despite the choice to focus on a single simulation, this simulation is not in the statistical steadystate and departure from stationarity is expected to play a role in particular in the parallel force balance Eq.(7). As the model derived in the previous section is strictly valid only for steady-state, a perfect match between the neoclassical flux given by the code and the theoretical prediction is not expected. Nevertheless, it turns out that the flux predicted by the model presented in section 2.3 gives the right order of magnitude for the impurity flux coming from GYSELA as will be discussed 
Figure 1: Radial variation of collisionality of the impurity (blue). The limit between plateau and Pfirsch-Schlüter regime is represented by the black line

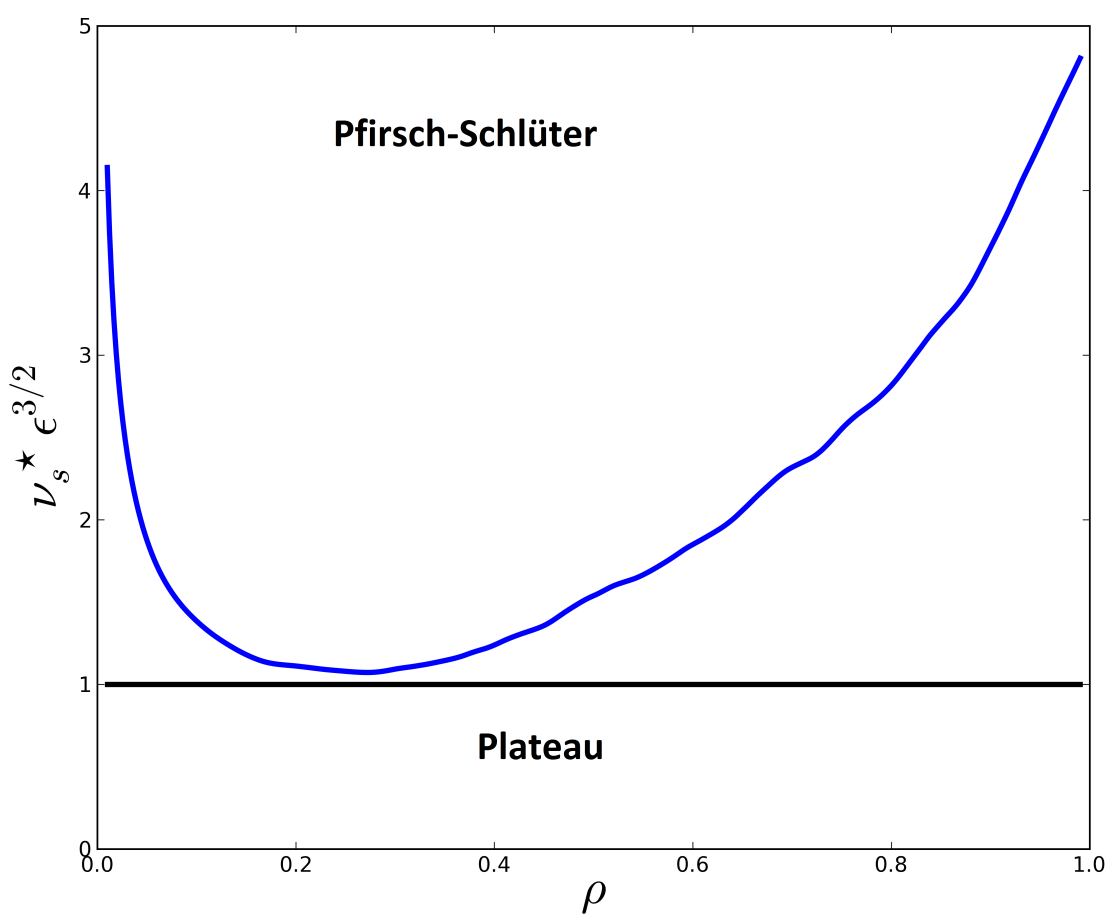

hereafter. Note that the cost of this single simulation is approximately 3 millions of CPU hours. This cost did not allow a large scan of parameters.

\subsection{Definitions of fluxes}

The flux of particles coming from GYSELA does not make any difference between neoclassical and turbulent fluxes as both are treated self-consistently within the code. This observation raises the question of how to separate the two contributions a posteriori. For this purpose, the definitions proposed by Esteve et al. [7] are used:

$$
\begin{aligned}
\left\langle\Gamma_{s}^{n e o} \cdot \nabla \psi\right\rangle_{\psi} & =\left\langle\int d^{3} v \bar{f}_{s}\left(\boldsymbol{v}_{D, s}+\overline{\boldsymbol{v}}_{E}^{n=0}\right) \cdot \nabla \psi\right\rangle_{\psi} \\
\left\langle\Gamma_{s}^{\text {turb }} \cdot \nabla \psi\right\rangle_{\psi} & =\left\langle\int d^{3} v \bar{f}_{s} \overline{\boldsymbol{v}}_{E}^{n \neq 0} \cdot \nabla \psi\right\rangle_{\psi}
\end{aligned}
$$

where $\overline{\boldsymbol{v}}_{E}^{n=0}=\left\langle\overline{\boldsymbol{v}}_{E}\right\rangle_{\varphi}$ and $\overline{\boldsymbol{v}}_{E}^{n \neq 0}=\overline{\boldsymbol{v}}_{E}-\overline{\boldsymbol{v}}_{E}^{n=0}$. The definition of these fluxes is not unique and some authors call "turbulent" the flux associated with the $E \times B$ drift and "neoclassical" the flux associated with the magnetic field drift. These fluxes are defined as

$$
\begin{aligned}
& \left\langle\Gamma_{s}^{D} \cdot \nabla \psi\right\rangle_{\psi}=\left\langle\int d^{3} v \bar{f}_{s} \boldsymbol{v}_{D, s} \cdot \nabla \psi\right\rangle_{\psi} \\
& \left\langle\Gamma_{s}^{E} \cdot \nabla \psi\right\rangle_{\psi}=\left\langle\int d^{3} v \bar{f}_{s} \overline{\boldsymbol{v}}_{E} \cdot \nabla \psi\right\rangle_{\psi}
\end{aligned}
$$

Fig.2 depicts the different fluxes for the impurity in the simulation presented in the previous section. There is a clear difference between the definitions of the turbulent and neoclassical fluxes (solid lines) and the definitions sometimes found in the literature (dotted lines). This observation means that the axisymmetric modes of the electric potential actively contribute to the impurity 
Figure 2: Radial variation of the impurity fluxes. These fluxes are time averaged on approximately $3000 \omega_{c i}$.

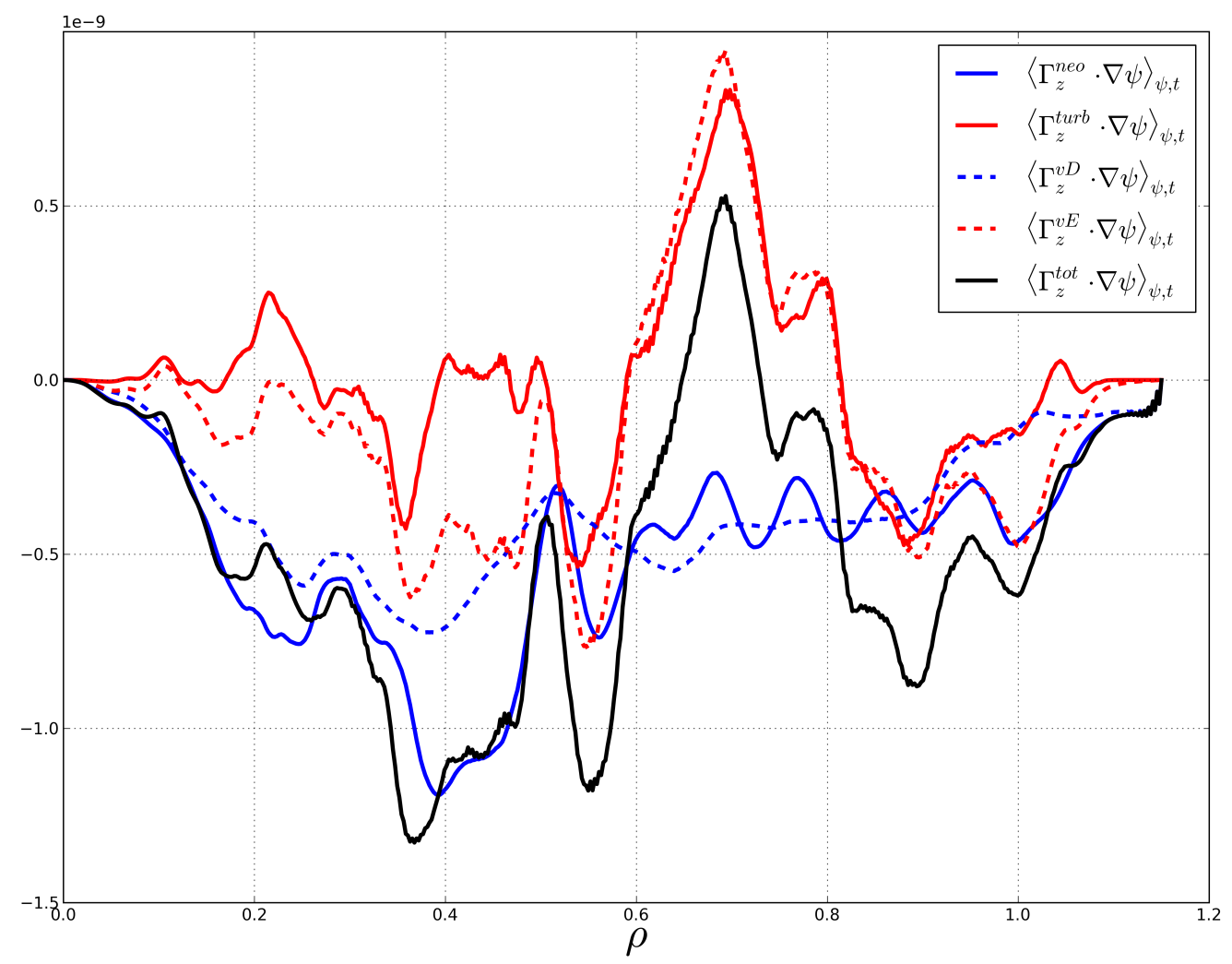

flux. Note also that turbulent and neoclassical fluxes are of the same order of magnitude, although sometimes of different signs. The overall transport of tungsten is mainly inward in this simulation.

For a fair comparison with the theoretical prediction of the neoclassical flux derived in the section 2 , it is mandatory to use the definition given in this thesis Eq.(18). This comparison is discussed in section 3.4 .

\subsection{Poloidal asymmetries and pressure anisotropy}

In the considered GYSELA simulation, the poloidal asymmetries are generated by turbulence. Indeed the only source used is a heat source with no poloidal dependence. The poloidal asymmetry of the tungsten density Fig.3 is of the order of $30 \%$ and can be expressed in the form [4]

$$
N_{z}=\left\langle N_{z}\right\rangle(\psi)[1+\delta(\psi) \cos \theta+\Delta(\psi) \sin \theta]
$$

The knowledge of $\delta(\psi)$ and $\Delta(\psi)$ then allows one to compute the impact of density asymmetries on the first and second terms of Eq.(17), which correspond to terms already present in the literature [4]. Indeed, one can show that these terms take the following simple expressions [4]:

$$
\begin{aligned}
\left\langle\frac{N_{z}}{B^{2}}\right\rangle_{\psi}-\frac{1}{\left\langle\frac{B^{2}}{N_{Z}}\right\rangle_{\psi}} & =\frac{\left\langle N_{z}\right\rangle_{\psi}}{\left\langle B^{2}\right\rangle_{\psi}}\left[2 \epsilon(\epsilon+\delta)+\frac{\delta^{2}+\Delta^{2}}{2}\right] \\
\left\langle N_{z}\right\rangle_{\psi}-\frac{\left\langle B^{2}\right\rangle_{\psi}}{\left\langle\frac{B^{2}}{N_{z}}\right\rangle_{\psi}} & =\left\langle N_{z}\right\rangle_{\psi}\left[\epsilon \delta+\frac{\delta^{2}+\Delta^{2}}{2}\right]
\end{aligned}
$$

In these expressions, the contribution of density poloidal asymmetry is contained only in the $\delta$ and $\Delta$ terms. Fig.4 shows the radial shape of Eq.(22) and Eq.(23) for the considered GYSELA simulation. It readily appears that both expressions remain close to their value at $\delta=\Delta=0$, 
Figure 3: Left: poloidal asymmetry of the impurity density. Right: reconstruction of the poloidal asymmetry with $\delta(\psi)$ and $\Delta(\psi)$
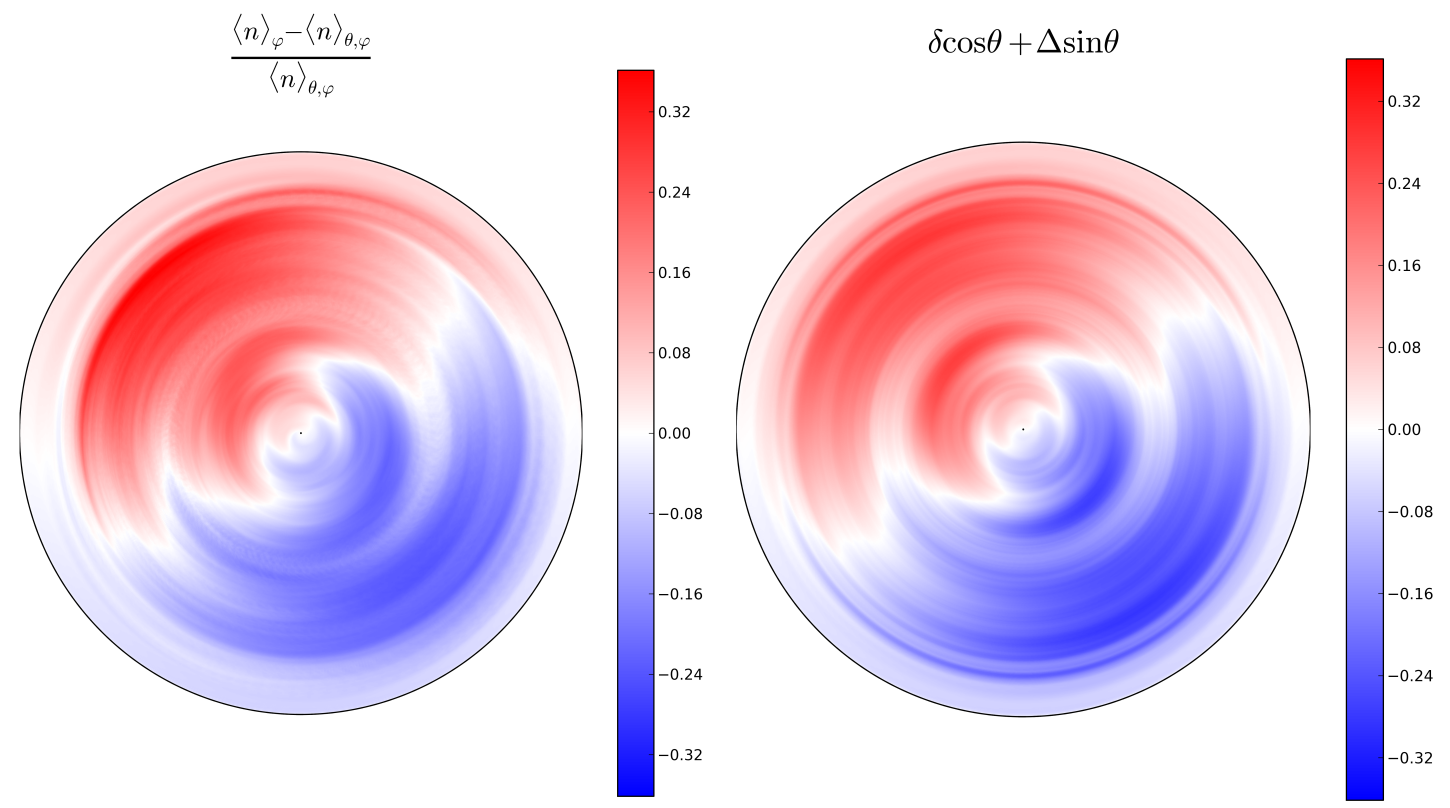

i.e. in the absence of any poloidal asymmetry. More precisely, the relative impact of the poloidal asymmetry of density compared with the one of the magnetic field is moderate in the deep core and weak in the outer part of the simulation.

Fig. 5 shows that the poloidal asymmetries of both parallel and perpendicular pressure are significant in the inner part of the simulation where the banana/plateau contribution is expected to be important $\left(0.15 \leq \frac{r}{a} \leq 0.4\right)$.

\subsection{Comparison between GYSELA results and theoretical predictions}

An important point for the comparison between the theoretical prediction and the results of the GYSELA code is the value of the screening factor. From the theoretical point of view, the PfirschSchlüter component of the screening factor should be $H_{\text {theo }}^{P S}=-\frac{1}{2}$. This can be seen in the expression of $\frac{1}{L_{\psi, i}}=\frac{\partial \ln P_{i}}{\partial \psi}-\frac{3}{2} \frac{\partial \ln T_{i}}{\partial \psi}=\frac{\partial \ln N_{i}}{\partial \psi}-\frac{1}{2} \frac{\partial \ln T_{i}}{\partial \psi}$. In this expression, there is a factor +1 coming from the difference of velocities $\left(\frac{\partial \ln P_{i}}{\partial \psi}\right)$ and a factor $-\frac{3}{2}$ coming from the main ion parallel heat flux $\left(-\frac{3}{2} \frac{\partial \ln T_{i}}{\partial \psi}\right)$. From the standard neoclassical theory, the main ion parallel heat flux is expected to be (Eq.(18) of $[8])$ :

$$
q_{\| i}=\frac{\left\langle B q_{\| i}\right\rangle_{\psi} B}{\left\langle B^{2}\right\rangle_{\psi}}-\frac{5}{2} \frac{N_{i} T_{i}}{e} \frac{\partial T_{i}}{\partial \psi} I(\psi)\left(\frac{1}{B}-\frac{B}{\left\langle B^{2}\right\rangle_{\psi}}\right)
$$

In the considered simulation, the parallel heat flux coming from GYSELA is not in agreement with the theoretical prediction Eq.(24). More precisely, the poloidal shape is in fair agreement with the theoretical prediction but the amplitude is approximately half the one predicted. The effect of this disagreement is to reduce drastically the thermal screening factor of the Pfirsch-Schlüter component $H_{G Y S}^{P S} \simeq 0$. This disagreement between the predicted parallel heat flux and the one coming from the code had already been observed in a pure neoclassical case [8] but its origin remains unclear. A remaining bug in the advection part of the code appears unlikely given the numerous tests and benchmarks already successfully performed [6], although it cannot be ruled out completly. Alternatively, the discrepancy could be due to some shortcomings of the theoretical prediction. In the rest of this section, two distinct predictions are then used for the Pfirsch-Schlüter contribution of the flux Eq.(17). The first one, labeled with $H=-0.5$, is the one coming from the theory, i.e. with $\frac{1}{L_{\psi, i}}=\frac{\partial \ln P_{i}}{\partial \psi}-\frac{3}{2} \frac{\partial \ln T_{i}}{\partial \psi}=\frac{\partial \ln N_{i}}{\partial \psi}-\frac{1}{2} \frac{\partial \ln T_{i}}{\partial \psi}$. The second, labeled $H=0$, is the same prediction but using 
Figure 4: Radial shape of Eq.(22) in red and Eq.(23) in green. The blue curve represents the radial shape of Eq.(22) in the absence of poloidal asymmetry of the impurity density, i.e. for $\delta=\Delta=0$.

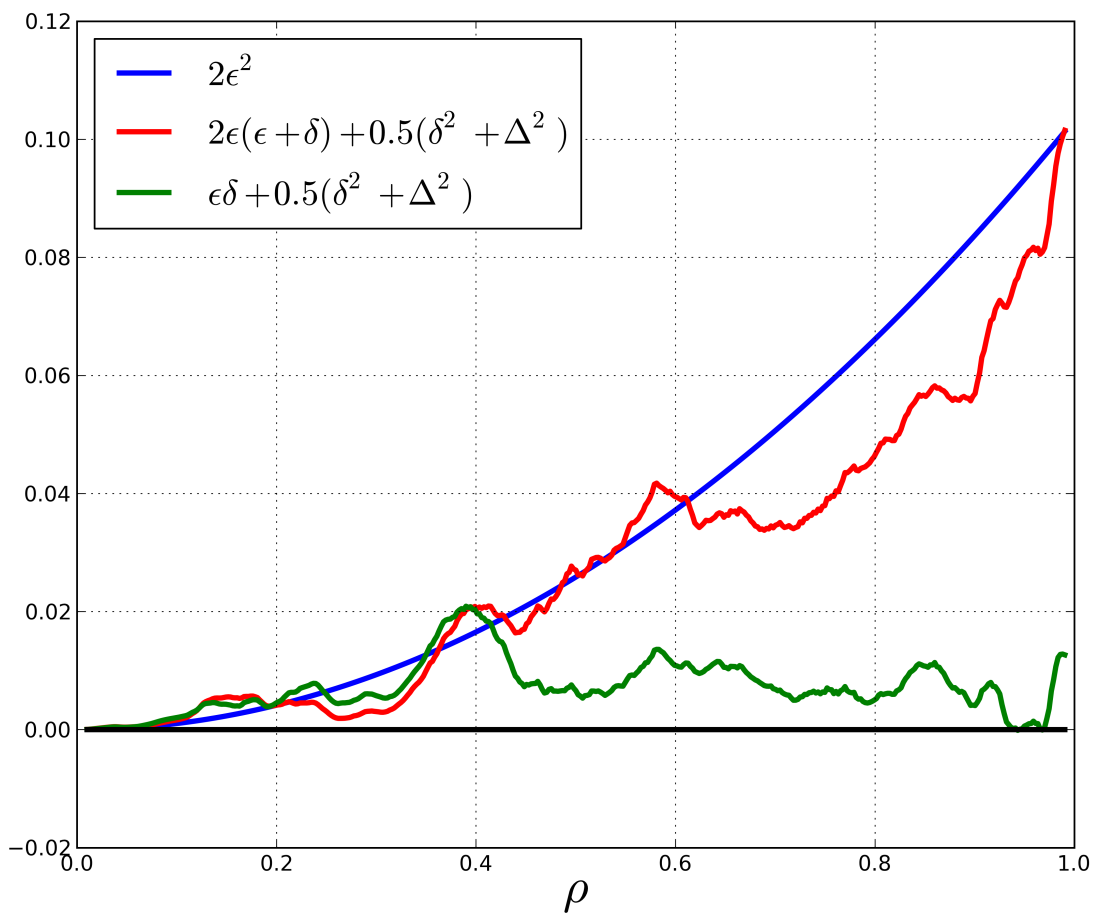

Figure 5: Left: parallel pressure. Middle: perpendicular pressure. Right: CGL tensor divided by the magnetic field.
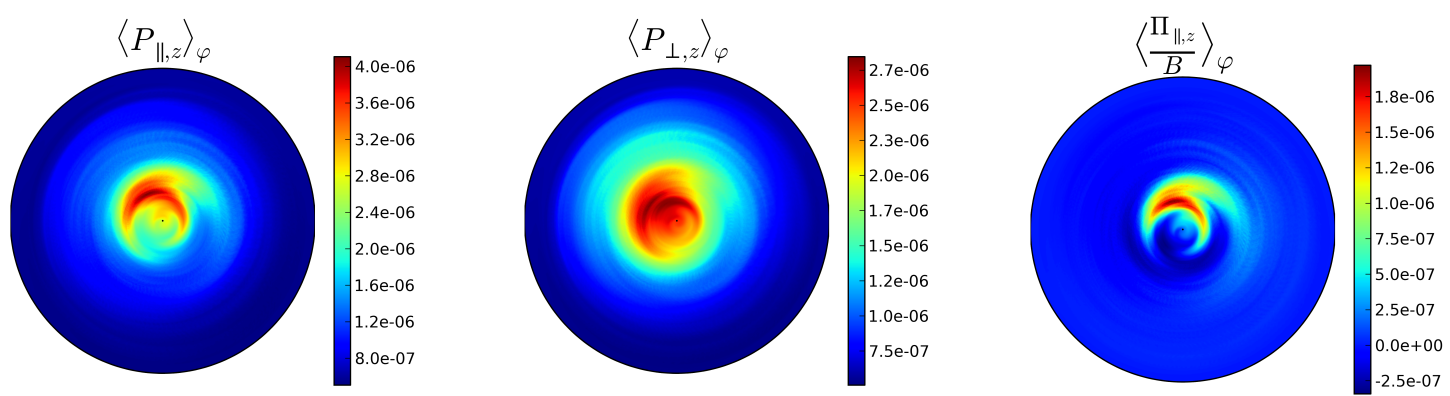
Figure 6: Components of the neoclassical flux. The banana/plateau contribution Eq.(16) is represented in green. The Pfirsch-Schlüter contribution Eq.(17) with $H=-\frac{1}{2}$ and $H=0$ are depicted respectively by the red and the blue curves. The various profiles, including their possible asymmetries, are taken from the GYSELA simulation.

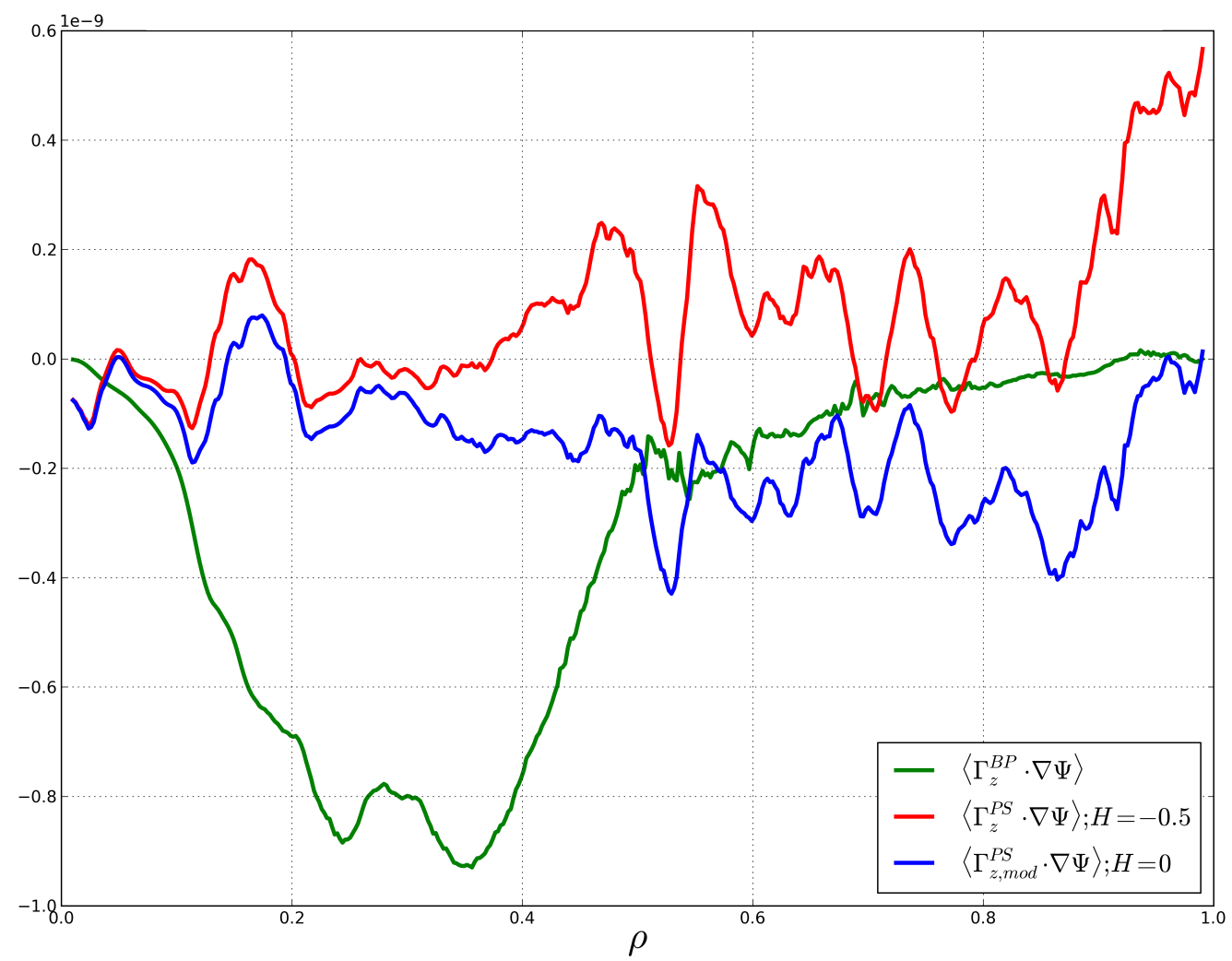

a different definition of the ion gradient contribution $\frac{1}{L_{\psi, i}}=\frac{\partial \ln P_{i}}{\partial \psi}-\frac{\partial \ln T_{i}}{\partial \psi}=\frac{\partial \ln N_{i}}{\partial \psi}$. This distinction makes a huge difference in the outer part of the simulation, as discussed in the following.

Fig. 6 shows the radial dependence of the banana/plateau contribution Eq.(16) (green curve) and the Pfirsch-Schlüter contribution Eq.(17) with the two definitions for $L_{\psi, i}$. The Pfirsch-Schlüter prediction with $H=-\frac{1}{2}$ is depicted in red and the one with $H=0$ is depicted in blue. From this figure, it is clear that the banana/plateau contribution is large where the impurity is close to the plateau regime $\left(0.15 \leq \frac{r}{a} \leq 0.4\right)$ as expected . For the Pfirsch-Schlüter component, there is a large impact of the screening factor, especially in the outer part of the simulation.

The final test consists in comparing the neoclassical flux coming from GYSELA with the neoclassical prediction Eq.(15). The definition of the neoclassical flux is given in Eq.(18).The comparison between the GYSELA results (black curve) and the theoretical prediction (red and blue curves) is depicted on Fig.7. The right order of magnitude is recovered with a vanishing thermal screening $H=0$ (blue curve) as expected from the previous discussion.

\section{Generation of poloidal asymmetries}

In experiments, the poloidal asymmetries are often induced by external sources via temperature anisotropies or high Mach numbers. A way to appreciate how heating systems can generate poloidal asymmetries is to consider the following formula [3, 10]:

$$
\frac{N_{z}(\psi, \theta)}{N_{z}^{\star}(\psi)}=\frac{T_{\perp, z}(\psi, \theta)}{T_{\perp, z}^{\star}(\psi)} \exp \left\{-\frac{e Z\left[\phi(\psi, \theta)-\phi^{\star}(\psi)\right]}{T_{\|, z}(\psi)}+\frac{m_{z} \Omega_{z}^{2}}{2 T_{\|, z}(\psi)}\left[R^{2}(\psi, \theta)-R^{\star 2}(\psi)\right]\right\}
$$

where the quantities with a star are taken at the outer mid-plane $f^{\star}(\psi)=f(\psi, \theta=0)$. In this formula, a rigid body like rotation is assumed for the impurity $\Omega_{z}$. In Eq.(25), the poloidal asymmetry 
Figure 7: Radial comparison of the theoretical predictions of the neoclassical radial impurity flux ( $H=-\frac{1}{2}$ in red, $H=0$ in blue) with the neoclassical flux coming from the GYSELA code (black).

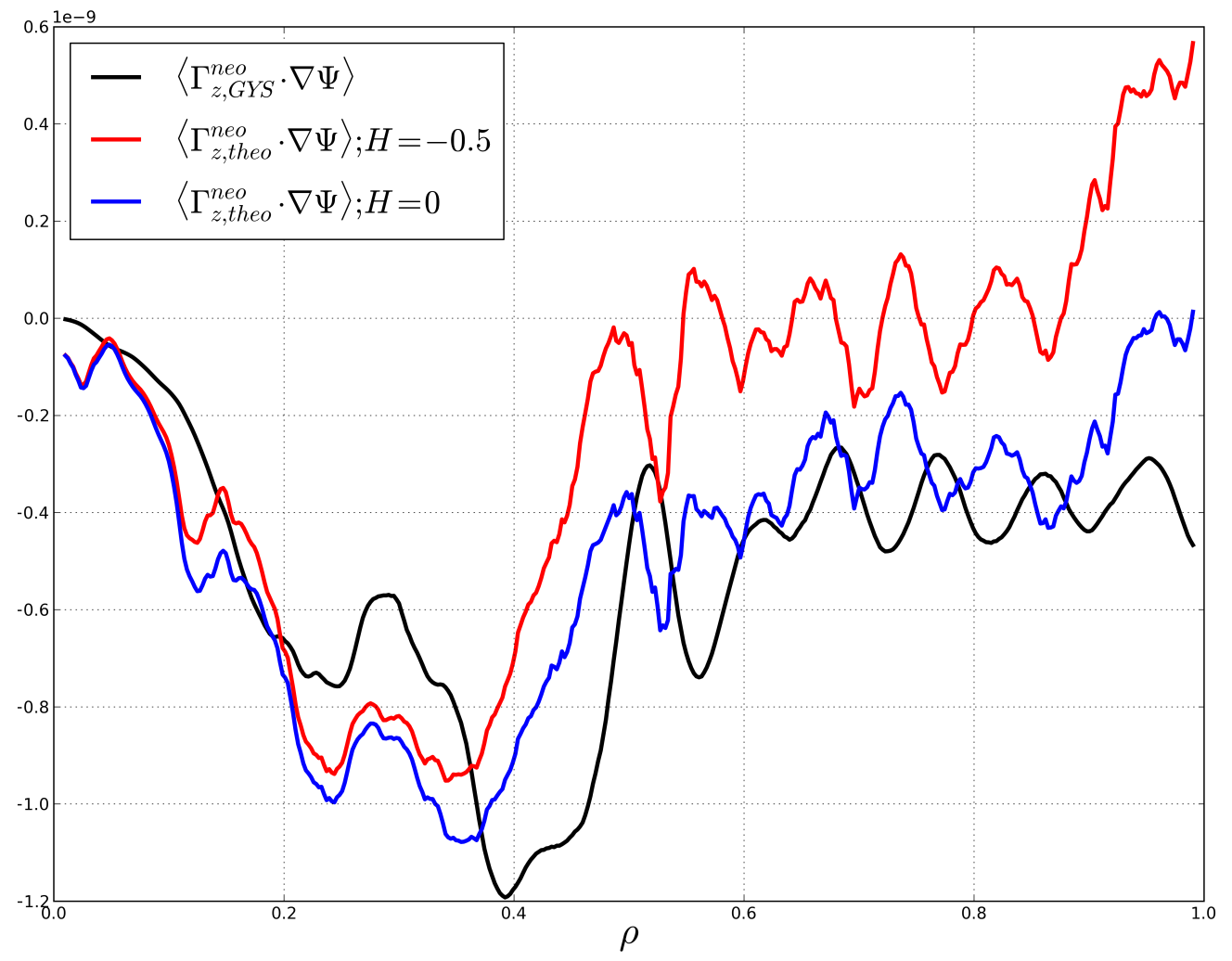

of the perpendicular temperature is associated with anisotropy.

$$
\frac{T_{\perp, z}(\psi, \theta)}{T_{\perp, z}^{\star}(\psi)}=\left[\frac{T_{\perp, z}^{\star}(\psi)}{T_{\|, z}(\psi)}+\left(1-\frac{T_{\perp, z}^{\star}(\psi)}{T_{\|, z}(\psi)}\right) \frac{B^{\star}(\psi)}{B(\psi, \theta)}\right]
$$

Eq. (25) clearly shows that toroidal rotation can generate poloidal asymmetry. This is in particular the case when Neutral Beam Injection (NBI) is used for current generation [15]. The combination of Eq.(25) and Eq.(26) indicates that anisotropic heating like the one provided by Ion Cyclotron Resonance Heating (ICRH) can also produce a poloidal asymmetry [16, 17].

In the simulation presented in the previous section, an isotropic heat source with no poloidal asymmetry has been used. According to Eq.(25) and Eq.(26), no poloidal asymmetry is expected from this source. Moreover, neoclassical physics predicts weak poloidal asymmetries [12, 13]. The large poloidal asymmetries observed in section 3.3 are therefore induced by turbulence.

From Eq.(25), it is clear that even weak poloidal asymmetries of the electric field can lead to significant poloidal asymmetries for heavy impurities due to their large charge number $Z$. Fig.8 shows that in the considered simulation, the poloidal asymmetry of the electric potential is of the order of $\frac{e\left(\phi_{n=0}-\phi_{m=0, n=0}\right)}{T} \sim 1 \%$ where $m$ and $n$ are respectively the poloidal and toroidal mode numbers. As a reminder, the amplitude of the density asymmetry for the impurity in the considered simulation is of the order of $30 \%$ (Fig.3). As the charge of the impuriy is $Z=40$, the right order of magnitude of poloidal asymmetry of the impurity density is retrieved with this simple argument. This was already observed in a previous study with the code GYSELA [7].

To quantify the relationship between electric potential and impurity poloidal asymmetries, a numerical test has been done. The same simulation as the one presented in the previous section has been performed except that a numerical filter removing all axisymmetric modes of the potential with poloidal asymmetries $(m \neq 0, n=0)$ has been applied at each time step. Fig.9 depicts the poloidal asymmetry of the impurity density in both cases using the following definitions $N_{z}=\left\langle N_{z}\right\rangle(\psi)[1+\delta(\psi) \cos \theta+\Delta(\psi) \sin \theta]$. From this figure, it is clear that the poloidal asymmetry of the electric field has an impact on the poloidal asymmetry of the impurity density. One should 
Figure 8: Amplitude of the non zonal electric potentital in the considered GYSELA simulation

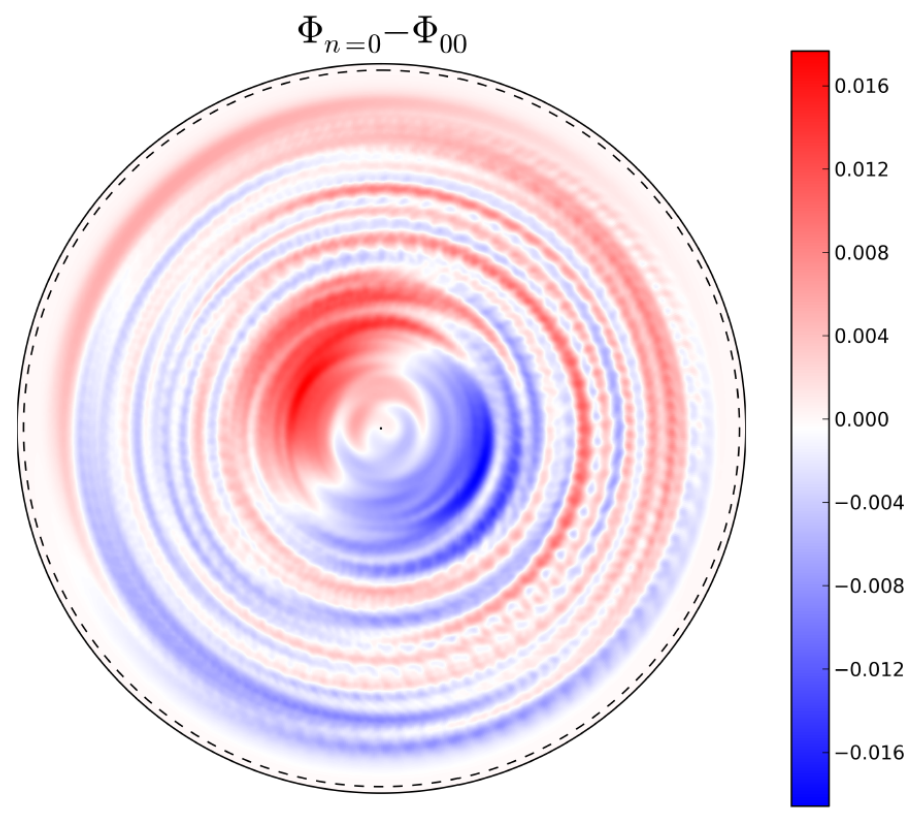

note that there are still poloidal asymmetries of the impurity density when the axisymmetric part of the electric field exhibits no poloidal asymmetry.

To understand how turbulence can generate poloidal asymmetries, one can use the Vlasov equation for the axisymmetric components neglecting particle trapping reads (Eq.(3) of [5]):

$$
-i\left(\Omega-M \Omega_{t}\right) \bar{g}_{M, \Omega}+\Omega_{D z}\left(\bar{g}_{M+1, \Omega}-\bar{g}_{M-1, \Omega}\right)=-i \Omega \frac{F_{e q, z}}{T_{e q, z}} e Z \bar{\phi}_{M, \Omega}-\left[\tilde{\overline{\boldsymbol{v}}}_{E} \cdot \nabla \tilde{\bar{g}}\right]_{M \Omega}
$$

where $\Omega$ is the frequency of the mode which is considered, $\Omega_{t}=\frac{v_{\|}}{q R_{0}}$ is the transit frequency of passing particles, $\Omega_{D z}=\frac{K_{r} \rho_{z} v_{T z}}{4 R_{0}}\left(2 \frac{v_{\|}^{2}}{v_{T z}^{2}}+\frac{v_{\perp}^{2}}{v_{T z}^{2}}\right)$ is the frequency associated with the magnetic drift, $\bar{g}$ is the non adiabatic part of the perturbed distribution function of the impurities. The tilde stands for the non axisymmetric component, i.e. $\tilde{\bar{g}}=\bar{g}-\langle\bar{g}\rangle_{\varphi}$. Here $M$ designates the poloidal mode number of the axisymmetric mode $(n=0)$ which is considered. It is clear from Eq.(27) that a poloidal asymmetry of the distribution function, for instance $\bar{g}_{1, \Omega}$ can be pumped by the zonal component $M=0$ due to the compressibility of the flow or directly generated by a poloidal asymmetry of the turbulent Reynolds stress $\left[\tilde{\overline{\boldsymbol{v}}}_{E} \cdot \nabla \tilde{\bar{g}}\right]_{M \Omega}$ due to turbulence ballooning $(M \neq 0)$. It is shown in $[5]$ that the pumping by the zonal component is more efficient for large frequencies and gives a $\sin \theta$ dependence for the $M= \pm 1$ mode. On the other hand, the generation of the poloidal asymmetry by the ballooning of the turbulent Reynolds stress dominates for lower frequencies and gives a $\cos \theta$ dependence.

In current tokamaks, poloidal asymmetries are often externally imposed by the use of NBI and/or ICRH systems. But the importance of the turbulent generated poloidal asymmetries over the one externally imposed should increase with the size of the machine. Moreover, ITER will use mainly ECRH system which are known to produce less poloidal asymmetries. Therefore, the turbulent generation of poloidal asymmetry, presented in this section, could be competitive or even dominant in next step devices.

Note that turbulence can also modify the Pfirsch-Schlüter contribution of the impurity flux Eq.(17) via the modification of $u$ Eq.(12). Indeed, $u$ is directly tied to the main ion poloidal rotation. This rotation can differ from its neoclassical prediction due to the presence of turbulence [18]. In the simulation considered in the previous section, this effect is small due to the small prefactor in front of the $u$ contribution Eq.(23) as shown in Fig.4. 
Figure 9: Comparison of density poloidal asymmetry with (solid line) or without (dotted line) poloidal asymmetry of electric potential. A time average on several turbulence correlation time has been performed.

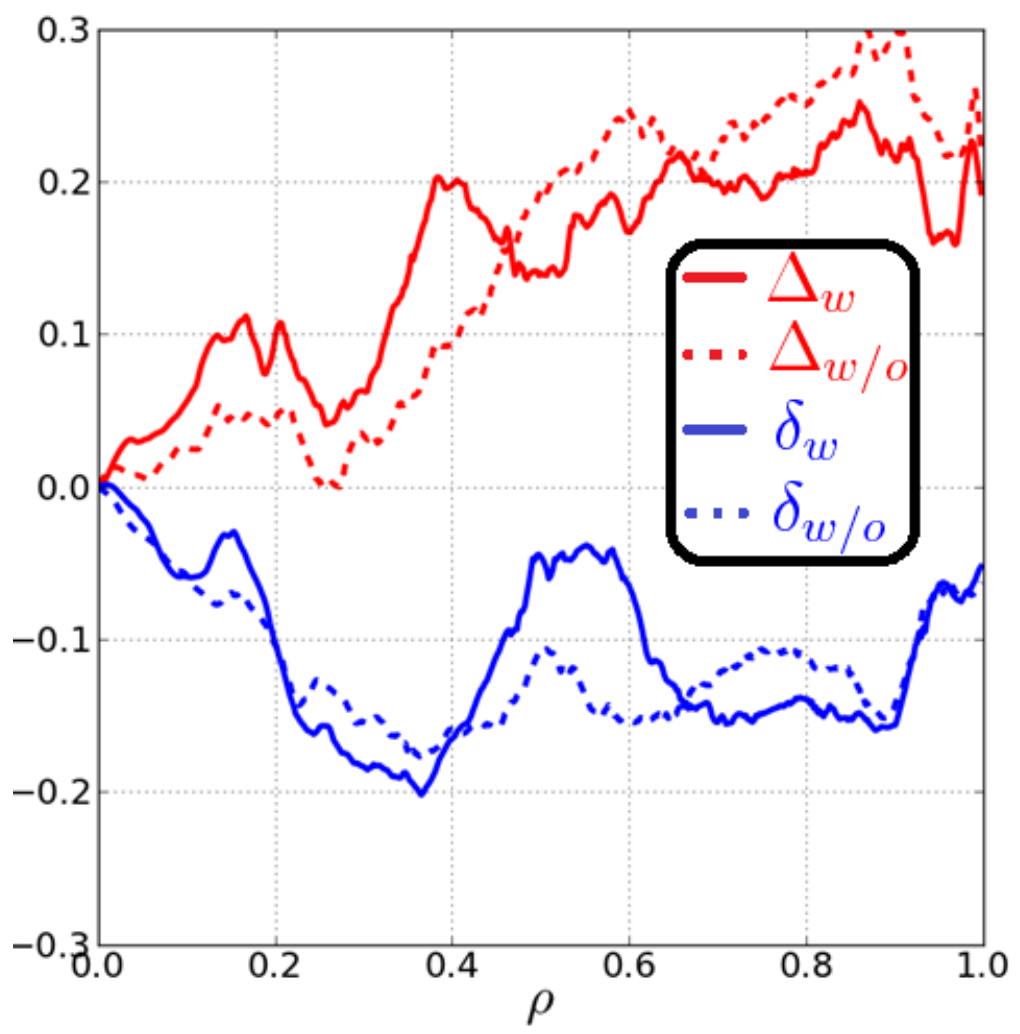

\section{Conclusion}

The impact of both poloidal asymmetry and anisotropy of impurity pressure on the neoclassical impurity flux has been derived analytically. These corrections can reveal important when considering realistic cases, where pressure poloidal asymmetry and anisotropy can be generated by turbulence and/or sources. In particular, accounting for these corrections to the standard neoclassical prediction could reveal critical in the perspective of impurity control by external heating systems.

From the theoretical stand point, this work provides a clear mechanism for synergy between neoclassical and turbulent processes in the context of impurity transport. Importantly, these predictions are found in fair agreement with a dedicated and highly resolved GYSELA simulation featuring both turbulent and neoclassical transports, where the correction terms appear to have a major contribution.

\section{Acknowledgments}

This work was granted access to the national HPC resources of OCCIGEN/CINES and European machine dedicated to fusion Marconi/CINECA. This work has been carried out within the framework of the EUROfusion Consortium and has received funding from the Euratom research and training program 2014-2018 under grant agreement No 633053 for the project WP17-ENR-CEA-02. This work was also supported by the Energy oriented Center of Excellence (EoCoE), grant agreement number 676629, funded within the Horizon2020 framework of the European Union. The views and opinions expressed herein do not necessarily reflect those of the European Commission. 


\section{Appendix: Derivation of the collisional parallel momentum exchange for trace and heavy impurities}

The goal of this appendix is to derive Eq.(11) and Eq.(12). The derivation begins with the expression of the collisional exchange of momentum in the case of trace $\left(\frac{N_{z} Z^{2}}{N_{i}} \ll 1\right)$ and heavy impurity $\left(\frac{m_{z}}{m_{i}} \gg 1\right):$

$$
R_{\| z i}=-N_{z} m_{z} \nu_{z i}\left[V_{\| z}-V_{\| z}+\frac{3}{5} \frac{q_{\| i}}{N_{i} T_{i}}\right]
$$

Moreover, the parallel velocity of any species can be expressed in the form

$$
V_{\| s}=\frac{K_{s}}{N_{s}} B-\frac{I \Omega_{s}}{B}
$$

with $\Omega_{s}=\frac{\partial \phi}{\partial \psi}+\frac{1}{N_{z} e Z} \frac{\partial P_{\perp s}}{\partial \psi}$ for any species $s$. In the same spirit, the parallel heat flux of the main ion takes the form

$$
\frac{q_{\| i}}{N_{i} T_{i}}=L_{i} B-\frac{5}{2} \frac{I}{e B} \frac{\partial T_{i}}{\partial \psi}
$$

where $L_{i}$ is a flux function. Substituting Eq.(29) and Eq.(30) in Eq.(28), one gets directly Eq.(11)

$$
R_{\| z i}=m_{z} \nu_{z i}\left\{-N_{Z} \frac{T_{i}}{e B} \frac{I}{L_{\psi}}+B\left(N_{z} u-K_{z}\right)\right\}
$$

at the condition to define $\frac{1}{L_{\psi}}=\frac{1}{L_{\psi, i}}+\frac{1}{L_{\psi, z}}$ with $\frac{1}{L_{\psi, i}}=\frac{\partial \ln P_{i}}{\partial \psi}-\frac{3}{2} \frac{\partial \ln T_{i}}{\partial \psi}$ and $\frac{1}{L_{\psi, z}}=-\frac{1}{T_{i} Z N_{z}} \frac{\partial P_{\perp z}}{\partial \psi}$ and

$$
u=\frac{K_{i}}{N_{i}}-\frac{3}{5} L_{i}
$$

The second step of this appendix is to prove the expression of $u$ Eq.(12). The first step is to compute $\frac{K_{i}}{N_{i}}$, to do so we use the vector expression of the ion velocity

$$
\boldsymbol{V}_{i}=\frac{K_{i}}{N_{i}} \boldsymbol{B}-\Omega_{i} R^{2} \nabla \varphi
$$

The poloidal velocity of the main ion is kown in the neoclassical theory $v_{\theta}=k_{n e o}\left(\nu_{\star}, \epsilon\right) \frac{\nabla T}{e B}$. The poloidal projection of Eq.(31) then leads to

$$
\frac{K_{i}}{N_{i}}=k_{n e o} \frac{I}{e\left\langle B^{2}\right\rangle_{\psi}} \frac{\partial T_{i}}{\partial \psi}
$$

$L_{i}$ can be computed directly with Eq.(24)

$$
L_{i}=\frac{\left\langle B \frac{q_{\| i}}{N_{i} T_{i}}\right\rangle_{\psi}}{\left\langle B^{2}\right\rangle_{\psi}}-\frac{5}{2} \frac{I}{e\left\langle B^{2}\right\rangle_{\psi}} \frac{\partial T_{i}}{\partial \psi}
$$

It has been showned [19] that $\left\langle B \frac{q_{\| i}}{N_{i} T_{i}}\right\rangle_{\psi} \sim \sqrt{\epsilon} L_{i}$. Then at lowest order in $\epsilon$, Eq.(12) is retrieved

$$
u=\left(k_{n e o}-\frac{3}{2}\right) \frac{I}{e\left\langle B^{2}\right\rangle_{\psi}} \frac{\partial T_{i}}{\partial \psi}
$$




\section{References}

[1] E. Joffrin, M. Baruzzo, M. Beurskens, C. Bourdelle, S. Brezinsek, J. Bucalossi, P. Buratti, G. Calabro, C.D. Challis, M. Clever, J. Coenen, E. Delabie, R. Dux, P. Lomas, E. de la Luna, P. de Vries, J. Flanagan, L. Frassinetti, D. Frigione, C. Giroud, M. Groth, N. Hawkes, J. Hobirk, M. Lehnen, G. Maddison, J. Mailloux, C.F. Maggi, G. Matthews, M. Mayoral, A. Meigs, R. Neu, I. Nunes, T. Puetterich, F. Rimini, M. Sertoli, B. Sieglin, A.C.C. Sips, G. van Rooij, I. Voitsekhovitch, and JET-EFDA Contributors. First scenario development with the jet new iter-like wall. Nuclear Fusion, 54(1):013011, 2014.

[2] T Pütterich, R Dux, R Neu, M Bernert, M N A Beurskens, V Bobkov, S Brezinsek, C Challis, J W Coenen, I Coffey, A Czarnecka, C Giroud, P Jacquet, E Joffrin, A Kallenbach, M Lehnen, E Lerche, E de la Luna, S Marsen, G Matthews, M-L Mayoral, R M McDermott, A Meigs, J Mlynar, M Sertoli, G van Rooij, the ASDEX Upgrade Team, and JET EFDA Contributors. Observations on the w-transport in the core plasma of jet and asdex upgrade. Plasma Physics and Controlled Fusion, 55(12):124036, 2013.

[3] R. Bilato, O. Maj, and C. Angioni. Modelling the influence of temperature anisotropies on poloidal asymmetries of density in the core of rotating plasmas. Nuclear Fusion, 54(7):072003, 2014 .

[4] C Angioni and P Helander. Neoclassical transport of heavy impurities with poloidally asymmetric density distribution in tokamaks. Plasma Physics and Controlled Fusion, 56(12):124001, 2014 .

[5] P. Donnel, X. Garbet, Y. Sarazin, Y. Asahi, F. Wilczynski, E. Caschera, G. Dif-Pradalier, P. Ghendrih, and C. Gillot. Turbulent generation of poloidal asymmetries of the electric potential in a tokamak. Plasma Physics and Controlled Fusion, 2018.

[6] V. Grandgirard, J. Abiteboul, J. Bigot, T. Cartier-Michaud, N. Crouseilles, G. Dif-Pradalier, Ch. Ehrlacher, D. Estève, X. Garbet, Ph. Ghendrih, G. Latu, M. Mehrenberger, C. Norscini, Ch. Passeron, F. Rozar, Y. Sarazin, E. Sonnendrucker, A. Strugarek, and D. Zarzoso. A 5d gyrokinetic full-f global semi-lagrangian code for flux-driven ion turbulence simulations. Computer Physics Communications, 207:35 - 68, 2016.

[7] D. Estève, Y. Sarazin, X. Garbet, V. Grandgirard, S. Breton, P. Donnel, Y. Asahi, C. Bourdelle, G. Dif-Pradalier, C. Ehrlacher, C. Emeriau, Ph. Ghendrih, C. Gillot, G. Latu, and C. Passeron. Self-consistent gyrokinetic modeling of neoclassical and turbulent impurity transport. Nuclear Fusion, 58(3):036013, 2018.

[8] P. Donnel, X. Garbet, Y. Sarazin, V. Grandgirard, Y. Asahi, N. Bouzat, E. Caschera, G. DifPradalier, C. Ehrlacher, P. Ghendrih, C. Gillot, G. Latu, and C. Passeron. A multi-species collisional operator for full-f global gyrokinetics codes: Numerical aspects and verification with the gysela code. Computer Physics Communications, 2018.

[9] G. Dif-Pradalier, G. Hornung, X. Garbet, Ph. Ghendrih, V. Grandgirard, G. Latu, and Y. Sarazin. The exb staircase of magnetised plasmas. Nuclear Fusion, 57(6):066026, 2017.

[10] F J Casson, C Angioni, E A Belli, R Bilato, P Mantica, T Odstrcil, T Pütterich, M Valisa, L Garzotti, C Giroud, J Hobirk, C F Maggi, J Mlynar, and M L Reinke. Theoretical description of heavy impurity transport and its application to the modelling of tungsten in jet and asdex upgrade. Plasma Physics and Controlled Fusion, 57(1):014031, 2015.

[11] M Romanelli and M Ottaviani. Effects of density asymmetries on heavy impurity transport in a rotating tokamak plasma. Plasma Physics and Controlled Fusion, 40(10):1767, 1998. 
[12] F. L. Hinton and Marshall N. Rosenbluth. Transport properties of a toroidal plasma at low-tointermediate collision frequencies. The Physics of Fluids, 16(6):836-854, 1973.

[13] F. L. Hinton and R. D. Hazeltine. Theory of plasma transport in toroidal confinement systems. Rev. Mod. Phys., 48:239-308, Apr 1976.

[14] T Fülöp and P Helander. Nonlinear neoclassical transport in a rotating impure plasma with large gradients. 6:3066-3075, 081999.

[15] C. Angioni, F. J. Casson, P. Mantica, T. Pütterich, M. Valisa, E. A. Belli, R. Bilato, C. Giroud, and P. Helander. The impact of poloidal asymmetries on tungsten transport in the core of jet h-mode plasmas. Physics of Plasmas, 22(5):055902, 2015.

[16] Ye O Kazakov, T Fülöp, I Pusztai, and T Johnson. Effect of plasma shaping and resonance location on minority ion temperature anisotropy in tokamak plasmas heated with icrh. Journal of Physics: Conference Series, 401(1):012011, 2012.

[17] M L Reinke, I H Hutchinson, J E Rice, N T Howard, A Bader, S Wukitch, Y Lin, D C Pace, A Hubbard, J W Hughes, and Y Podpaly. Poloidal variation of high- z impurity density due to hydrogen minority ion cyclotron resonance heating on alcator c-mod. Plasma Physics and Controlled Fusion, 54(4):045004, 2012.

[18] G. Dif-Pradalier, V. Grandgirard, Y. Sarazin, X. Garbet, and Ph. Ghendrih. Interplay between gyrokinetic turbulence, flows, and collisions: Perspectives on transport and poloidal rotation. Phys. Rev. Lett., 103:065002, Aug 2009.

[19] S Hirshman and D.J. Sigmar. Neoclassical transport of impurities in tokamak plasmas. 21:1079, 011981. 